\title{
A Search for Companions to L Dwarfs
}

\author{
P. R. Allen, D. W. Koerner, M. W. McElwain, G. R. Murphy \\ University of Pennsylvania, 209 S. 33rd St., Philadelphia, PA 19104 \\ I. Neill Reid, J. E. Gizis, and J. Davy Kirkpatrick \\ (STScI) (Univ. of Delaware) (IPAC)
}

\begin{abstract}
We present the results of two imaging surveys for companions to low-luminosity dwarfs, one with Keck to a limiting magnitude of $K=20$, and the other with HST to a limiting magnitude of $I=23$. No companions were found at separations of 0 " 5 to $20^{\prime \prime} 0$, but seven companions were found within $0 ! 5$. Based on sub-stellar evolutionary models, we estimate the companion detection efficiency as a function of mass ratio. This modeling, coupled with the data, place strong upper limits on the mass ratios of unobserved companions. We conclude that no companions exist in the optimal sensitivity regions of both surveys for $\mathrm{q}>0.85$ (HST), and $\mathrm{q}>0.4$ (Keck).
\end{abstract}

\section{Introduction}

Study of multiplicity enables a better understanding of star formation. The mass and separation distributions of companions are the fingerprints of star formation mechanisms. The multiplicity and distribution change as a function of spectral type. Duquennoy and Mayor (1991) find a $60 \%$ multiplicity for G dwarfs, while Fisher and Marcy (1992) find $40 \%$ for $\mathrm{M}$ dwarfs. With our surveys we will determine if this trend continues into the very low-mass regime.

\section{The Sample and Detections}

Targets for both samples were culled from the lists of known late-type dwarfs in the 2MASS and DENIS surveys. We have analyzed observations of 74 targets obtained with NIRC on Keck (Koerner et. al. 1999), and of 20 targets from WFPC2 on HST (Reid et. al. 2001), for a total of $\sim 90$ objects. Additionally, we have double or triple epoch data of the Keck sample to identify common proper motion. The Keck survey is sensitive to companions with $K<20$ for separations between $1^{\prime \prime} 0$ and $20{ }^{\prime \prime} 0$. The HST survey is sensitive to $I<23$ for separations between 0 ' 5 and $18^{\prime \prime} .2$. We are sensitive to near equal luminosity binaries, for Keck with $\Delta K \approx 0.1$ at separations $>0$ '! 3 , and for HST with $\Delta I<1.3$ at separations $>00^{\prime \prime} 07$. A total of seven companions were found in the combined sample at separations between 0.07 and 0.5 , with luminosity ratios near unity. There are no detections in our optimal sensitivity region. 

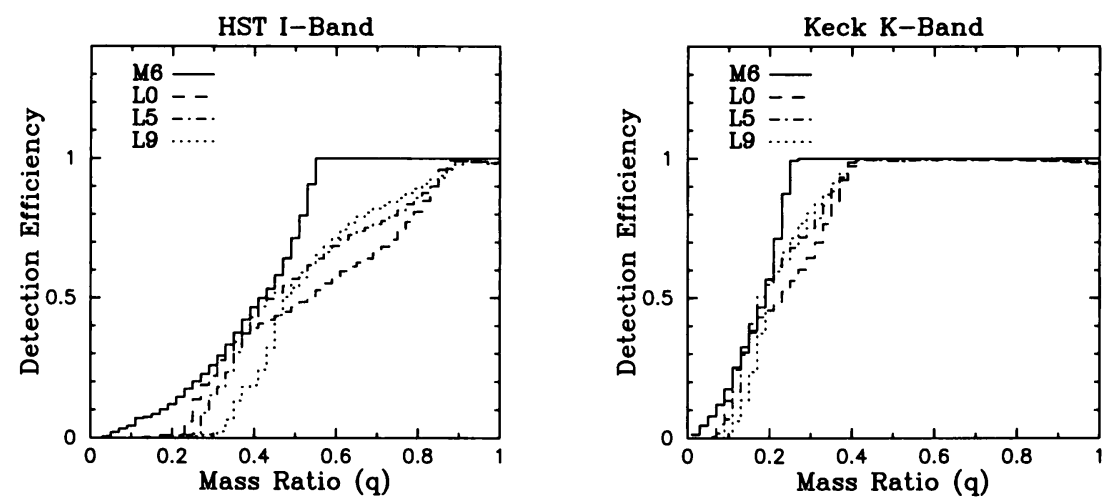

Figure 1. The modeled detection efficiency as a function of mass ratio for the HST (left) and Keck (right) surveys at an average distance of $30 \mathrm{pc}$. The curves represent four different spectral type primaries.

\section{Modeling the Companion Distribution}

In order to understand these null results, we constructed detection efficiency models based on the Burrows et. al. (2001) evolutionary models for low-mass objects. These models provide bolometric luminosity and effective temperature as a function of age. We transform these quantities into broad-band magnitudes, using observed bolometric corrections. It is necessary to derive an age distribution for each spectral type. The luminosities of sub-stellar objects are highly age dependent because they continually cool through many spectral classes at mass-dependent rates. The age distributions are estimated from a uniform star formation rate over $10 \mathrm{Gyrs}$. Given the primary's spectral type age distribution, we estimate the luminosity ratios of potential companions for each mass ratio.

To determine the detection efficiency, we apply the observed sensitivity limits to the models and determine the percentage of possible companions detectable for each mass ratio. The results of this calculation for four different primaries are shown in Figure 1. Based on these, we conclude that in the present sample of $\sim 90$ very low-mass primaries, no companions exist for $\mathrm{q}>0.85$ in separations between 0.5 and 18.2 for the HST $I$-band, and no companions exist for q $>0.4$ in separations between 1.0 and $20^{\prime \prime} 0$ for the Keck $K$-band. This implies that the trend of decreasing multiplicity with later spectral type continues into the very low-mass regime.

\section{References}

Burrows, A., Hubbard, W., Lunine, J., and Liebert, J., 2001, RvMP, 73, 719.

Duquennoy, A. and Mayor, M., 1991, A\&A, 248, 485.

Fischer, D. and Marcy, G., 1992, ApJ, 396, 178.

Koerner, D.W. et. al. 1999, ApJ, 526, L25.

Reid, I.N. et. al. 2001, ApJ, 121, 489. 